\title{
Flexural Properties of Wheat Straw Reinforced Polyester Composites
}

\author{
Chensong Dong*, Ian J. Davies
}

Department of Mechanical Engineering, Curtin University, GPO Box U1987, Perth, WA, 6845, Australia

\begin{abstract}
A study on the flexural properties of wheat straw reinforced polyester composites is presented in this paper. The wheat straw/polyester composites were made as per the hand layup procedure and the instructions provided by the manufacturers. These specimens were then tested in the three point bend configuration in accordance with ASTM D790-07 at a span to depth ratio of 16 . Four weight fractions of wheat straw $2 \%, 4 \%, 6 \%$ and $8 \%$ were chosen to be studied. The process-induced voids were studied and it is shown that voids play an important role in flexural properties. When voids exist, flexural modulus was calculated using a micromechanical model. It is shown from both the experimental results and calculation that flexural modulus decreases with the addition of wheat straw, and this reduction in flexural modulus is due to process-induced voids. Flexural strength of polyester resins does not increase with the addition of wheat straw as reinforcement. This is due to poor interfacial adhesion and the existence of voids. In order to achieve reinforcement, treatment of straw fibers and better processing techniques to reduce voids are needed.
\end{abstract}

Keywords Polymer-Matrix Composites (Pmcs), Wheat Straw, Flexural, Mechanical Testing

\section{Introduction}

The interest in natural fiber reinforced biocomposites has been growing rapidly during recent years, driven by increased environmental and health concerns, more sustainable methods of manufacture and reduced energy consumption. Natural fibers such as cotton, flax and hemp have been used as reinforcements in biocomposites[1]. Waste generation is a significant burden on the environment. Most food and forestry industry activities result in large amounts of by-products that are often treated as waste and sent to landfill. One of them is wheat straw. As a cheaper and more rapidly renewable source of cellulose-rich fibre, wheat straw has a great potential of being used as reinforcement in biocomposites.

It is shown from the literature that the research on wheat straw reinforced composites is quite limited[2-5]. Mishra and Sain[4] explored the advantages and commercial viability of using wheat straw as filler for thermoplastic composites. Wheat straw was investigated by Halvarsson et al.[5] as a raw material for manufacturing of medium density fibreboard (MDF) in a fully equipped pilot-plant. In this study, the flexural properties of wheat straw reinforced polyester composites are studied through a three-point bend test, in which a loading nose deflects a specimen at a set span and loading rate until fracture. A fixed span-to-depth

* Corresponding author:

chensong.dong@gmail.com (Chensong Dong)

Published online at http://journal.sapub.org/materials

Copyright (C) 2011 Scientific \& Academic Publishing. All Rights Reserved ratio 16 was used and four weight fractions of wheat straw $2 \%, 4 \%, 6 \%$ and $8 \%$ were chosen to be studied. The process-induced voids were also studied.

\section{Experiments}

The materials used in this study were wheat straw, SR250 orthophthalic polyester resin and MEKP catalyst (Fibreglass $\&$ Resin Sales Pty. Ltd.) Their properties are shown in Table 1[6]. Four weight fractions of wheat straw $2 \%, 4 \%, 6 \%$ and $8 \%$ were chosen to be studied. For reference, pure polyester specimens were also made and tested.

Table 1. Constituent materials and selected properties

\begin{tabular}{cccc}
\hline Material & $\begin{array}{c}\text { Tensile strength } \\
(\mathrm{MPa})\end{array}$ & $\begin{array}{c}\text { Tensile modulus } \\
(\mathrm{GPa})\end{array}$ & $\begin{array}{c}\text { Density } \\
\left(\mathrm{g} / \mathrm{cm}^{3}\right)\end{array}$ \\
\hline Wheat straw & 32 & 6.6 & 1.2 \\
Polyester & 52 & 4.0 & 1.26 \\
\hline
\end{tabular}

The wheat straw/polyester composites were made as per the hand layup procedure[7] and the instructions provided by the manufacturers. The mold being used in this study consists of a flat tray and a top plate. Prior to manufacturing, the mold surfaces were prepared by applying a layer of traffic wax and letting it dry for 20 to 25 minutes.

The required amount of unsaturated polyester resin was measured in a mixing cup. The suitable amount of MEKP catalyst was calculated according to the instruction provided by the manufacturer, i.e. $10 \mathrm{ml}$ of MEKP per kilogram of resin, and then added into the polyester resin. The polyester resin and MEKP catalyst were mixed for 2 minutes using the 
scrap-rotate-scrap mixing method as suggested by the manufacturer, where the sides and bottom of the mixing cup were scrapped in care by using a pop-stick to reduce trapping of air bubbles, while $90^{\circ}$ rotations were made.

The catalyzed polyester resin was then poured slowly into the tray at a distance of $30 \mathrm{~cm}$ above the tray, allowing extremely thin pouring stream to reduce trapping of air bubbles. The required amount of wheat straw was weighed and added to the mixture. The scrap-rotate-scrap method was again applied for 2 to 5 minutes or longer depending on the composition of wheat straw. The mixture was left untouched for 10 minutes to allow air bubbles to rise to the surface, and pop-stick was used to "pop" the bubbles.

The top plate was stacked onto the gel-like mixture and the mixture was left for 18 hours for curing. The composite was then post-cured for 2 hours at $80^{\circ} \mathrm{C}$ before being removed from the mold.

A finished composite part is shown in Figure 1. The finished composites were cut into test specimens of $25 \mathrm{~mm}$ wide using a diamond tipped circular saw blade. Testing was conducted in a three point bend configuration in accordance to procedure A of ASTM D790-07, using an Instron 550R universal testing machine at a span-to-depth ratio of 16 , as shown in Figure 2. The average loading rate for this analysis was in the order of $5 \mathrm{~mm} / \mathrm{min}$. For each specimen, its length, width, thickness and weight were measured in order to determine the density.

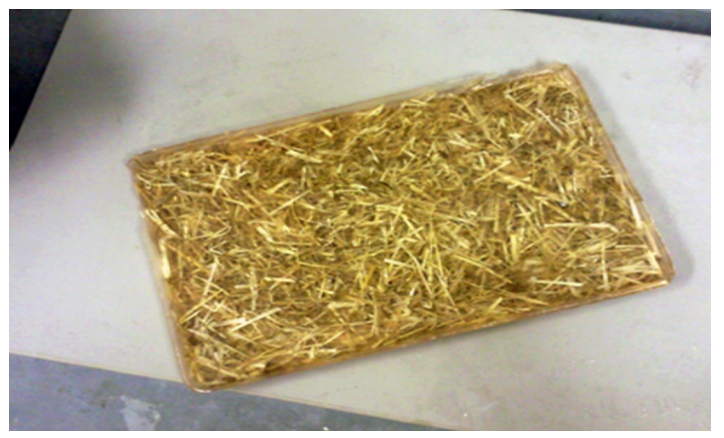

Figure 1. A finished wheat straw/polyester composite part

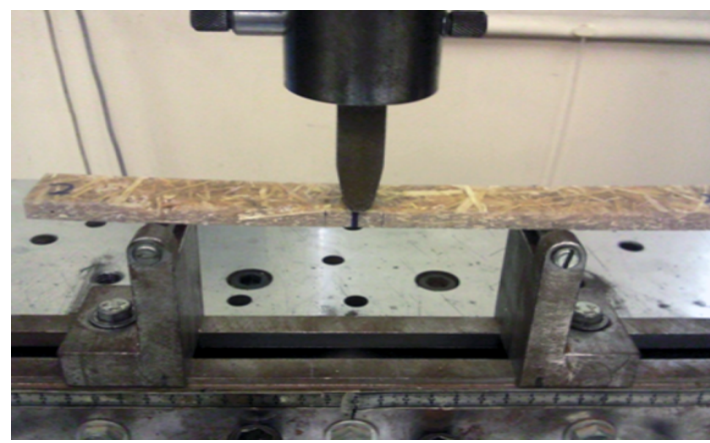

Figure 2. Flexural testing being conducted

From the load-deflection data obtained from testing, flexural strength $\left(\sigma_{F}\right)$, modulus $\left(E_{F}\right)$ and strain to failure $\left(\varepsilon_{F}\right)$ can be calculated by $[8]$ :

$$
\sigma_{F}=\frac{3 P_{\max } L}{2 b h^{2}}\left[1+6\left(\frac{D}{L}\right)^{2}-4\left(\frac{h}{L}\right)\left(\frac{D}{L}\right)\right]
$$

$$
\begin{gathered}
E_{F}=\frac{m L^{3}}{4 b h^{3}} \\
\varepsilon_{F}=\frac{6 D h}{L^{2}}
\end{gathered}
$$

where $L, b$ and $h$ are the span, width and depth of the specimen, $m$ is the slope of the tangent to the initial straight-line portion of the load-deflection curve, $D$ is the maximum deflection before failure, and $P_{\max }$ is the maximum load encountered before failure.

For each weight loading, 5 tests were conducted for each configuration, and the average values and variations were determined and presented. Following the testing, specimens were also inspected under an optical microscope in order to investigate any anomalies in flexural performance.

\section{Results and Discussion}

\subsection{Fracture Surface}

The micrograph images of representative failed specimens are shown in Figure 3. It is seen that all test specimens failed in a brittle manner. These images show that the matrix does not embedded with wheat straw fillers, i.e. fiber pullout and fracture, and voids exist. Poor adhesion of the fiber to the matrix and fiber breakages are some of the major factors that have been reported to be the main cause of failure in natural fiber composites[9]. Voids and fiber pull out result in the reduction of flexural, tensile and impact strength[2].



(a)

(b)

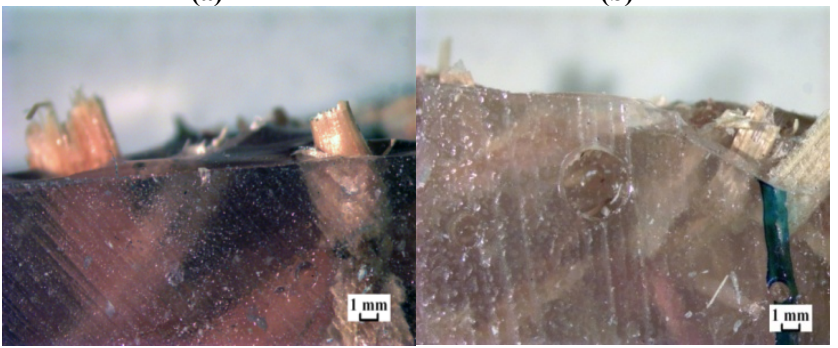

(c)

(d)

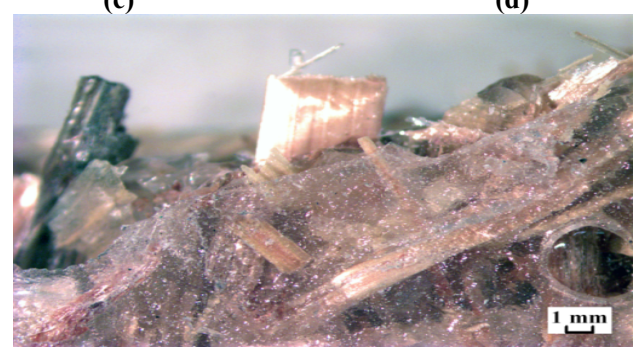

(e)

Figure 3. Micrograph images of failed specimens (a) pure polyester (b) $2 \%$ wheat straw (c) $4 \%$ wheat straw (d) $6 \%$ wheat straw (e) $8 \%$ wheat straw 


\subsection{Load-Displacement Curve}

The load-displacement curves from testing are shown in Figure 4. It is seen that load increases linearly with displacement until the failure point.

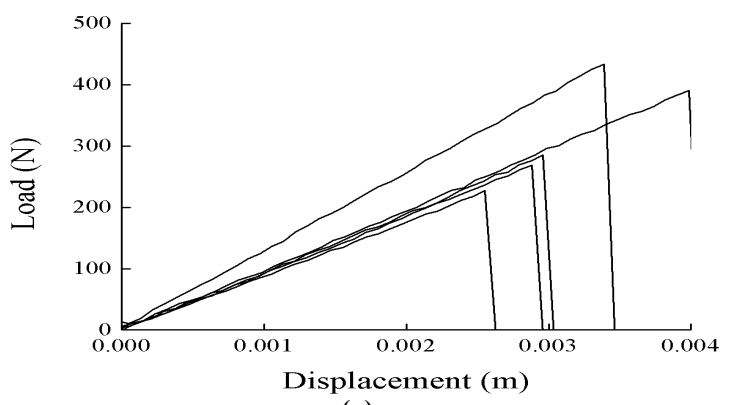

(a)

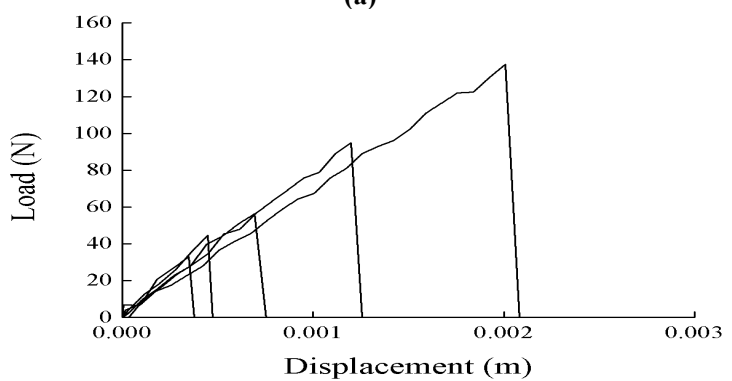

(b)

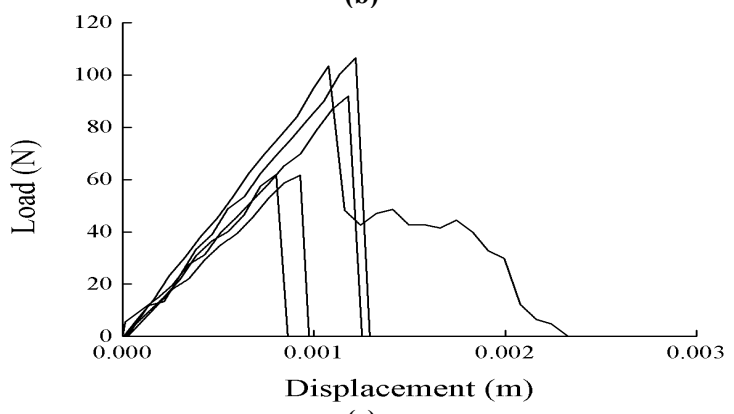

(c)

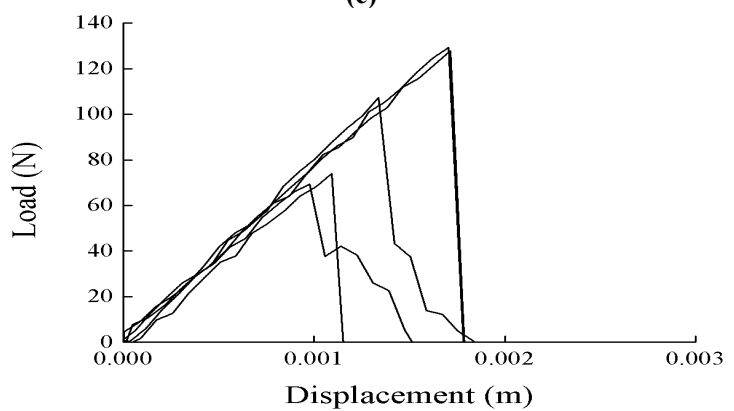

(d)

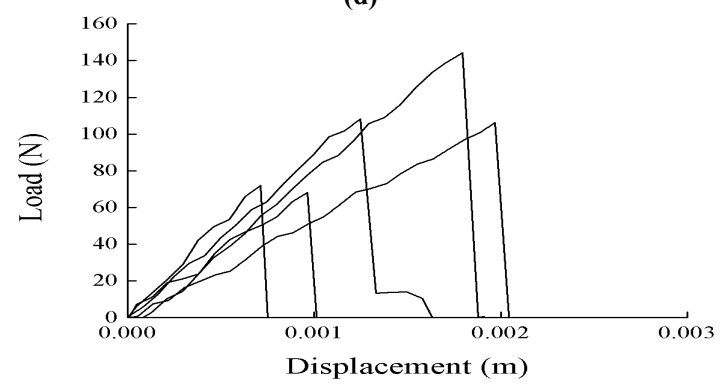

(e)

Figure 4. Load-displacement curves from flexural tests (a) pure polyester (b) $2 \%$ wheat straw (c) $4 \%$ wheat straw (d) $6 \%$ wheat straw (e) $8 \%$ wheat straw

\subsection{Void Content}

When voids are present, the fiber weight fraction $w_{f}$ is given by

$$
w_{f}=\frac{\rho_{f} V_{f}}{\rho_{f} V_{f}+\rho_{m}\left(1-V_{f}-V_{v}\right)}
$$

where $\rho_{c}, \rho_{f}$ and $\rho_{m}$ are the densities of composite, fiber and matrix, respectively, and $V_{f}$ and $V_{v}$ are the volume fractions of fiber and voids, respectively.

If the fiber weight fraction is known, the fiber volume fraction can be found by

$$
V_{f}=\frac{1-V_{v}}{1+\frac{\rho_{f}}{\rho_{m}}\left(\frac{1}{w_{f}}-1\right)}
$$

The density of composite can be obtained using the rule of mixtures as given below.

$$
\rho_{c}=\rho_{f} V_{f}+\rho_{m}\left(1-V_{f}-V_{v}\right)
$$

In this study, the void contents in the pure polyester specimens were unknown. Thus, the pure polyester specimens were regarded as void-free and the voids caused by the introduction of wheat straw were studied. The void contents were derived from the fiber weight fractions and densities using Eqns. 5 and 6. The densities and the void contents obtained are shown in Table 2. It is seen that void content increases with weight percentage of wheat straw.

Table 2. Measured thicknesses, densities and void contents

\begin{tabular}{cccc}
\hline $\begin{array}{c}\text { Weight percent- } \\
\text { age of wheat straw }\end{array}$ & $\begin{array}{c}\text { Average thick- } \\
\text { ness }(\mathrm{mm})\end{array}$ & $\begin{array}{c}\text { Measured den- } \\
\text { sity }\left(\mathrm{g} / \mathrm{cm}^{3}\right)\end{array}$ & $\begin{array}{c}\text { Void con- } \\
\text { tent }(\%)\end{array}$ \\
\hline 0 & 8.20 & 1.260 & 0 \\
2 & 8.63 & 1.169 & 7.13 \\
4 & 8.67 & 1.147 & 8.79 \\
6 & 8.92 & 1.132 & 9.89 \\
8 & 9.49 & 1.114 & 11.23 \\
\hline
\end{tabular}

\subsection{Flexural Modulus}

Graphs and other numbered figures should appear throughout the text as close to their mention as possible. Figures shouldn't infringe upon the page borders.

In order to take into account the effect of voids, the effective modulus of resin $E_{m e}$ was first calculated using Kerner's model[10], which is given by

$$
E_{m e}=\frac{9 K_{m e} G_{m e}}{3 K_{m e}+G_{m e}}
$$

where $G_{m e}$ and $K_{m e}$ are the shear and bulk moduli of resin, given by

$$
\begin{gathered}
G_{m e}=G_{m} \frac{\left(7-5 v_{m}\right)\left(1-\frac{V_{v}}{V_{m}}\right)}{\left(7-5 v_{m}\right)+\left(8-10 v_{m}\right) \frac{V_{v}}{V_{m}}} \\
K_{m e}=\frac{4 K_{m} G_{m}\left(1-\frac{V_{v}}{V_{m}}\right)}{4 G_{m}+3 K_{m} \frac{V_{v}}{V_{m}}}
\end{gathered}
$$


For a unidirectional short fiber composite, its longitudinal and transverse moduli $E_{11}$ and $E_{22}$ are given by Halpin-Tsai equations[11] as

$$
\begin{gathered}
E_{11}=\frac{1+2\left(l_{f} / d_{f}\right) \eta_{L} V_{f}}{1-\eta_{L} V_{f}} E_{m e} \\
E_{22}=\frac{1+2 \eta_{T} V_{f}}{1-\eta_{T} V_{f}} E_{m e}
\end{gathered}
$$

where

$$
\begin{aligned}
& \eta_{L}=\frac{\left(E_{f} / E_{m e}\right)-1}{\left(E_{f} / E_{m e}\right)+2\left(l_{f} / d_{f}\right)} \\
& \eta_{T}=\frac{\left(E_{f} / E_{m e}\right)-1}{\left(E_{f} / E_{m e}\right)+2}
\end{aligned}
$$

$l_{f}$ and $d_{f}$ are average fiber length and diameter, respectively. In this study, an average fiber length-diameter ratio 10 was assumed.

For a randomly oriented short fiber composite, its elastic modulus is given by

$$
E_{\text {random }}=\frac{3}{8} E_{11}+\frac{5}{8} E_{22}
$$

Because of the linear load-displacement relationships, the elastic modulus and the flexural modulus are very close. The flexural moduli from the experiments and calculation are shown in Figure 5. It is seen that although some differences exist, both the experimental results and calculations show that flexural modulus decreases with the addition of wheat straw, and this reduction in flexural modulus is due to process-induced voids.

\subsection{Flexural Strength}

The flexural strengths from the experiments are shown in Figure 6 . It is shown that flexural strength decreases rapidly with the addition of wheat straw. The reason is possibly hydrophilic wheat straw is incompatible with hydrophobic polyester. This will result in poor interfacial adhesion. In addition, the existence of voids causes decreased flexural strength. In order to achieve reinforcement, treatment of straw fibers and better processing techniques to reduce voids are needed.

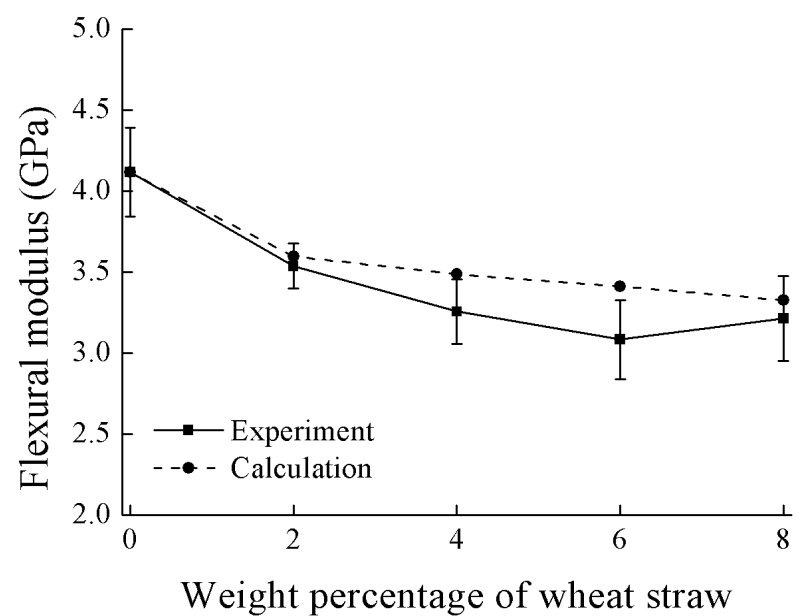

Figure 5. Flexural moduli from experiments and calculation

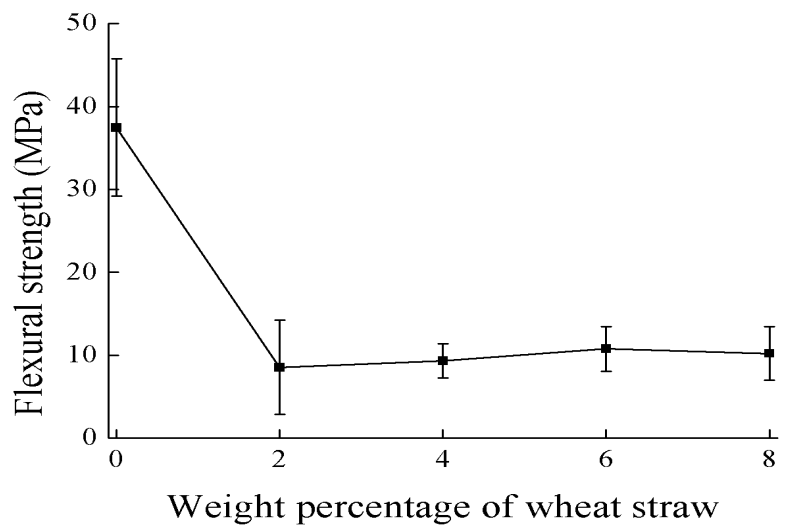

Figure 6. Flexural strength vs. weight percentage of wheat straw

\subsection{Strain to Failure}

The strains to failure from the experiments are shown in Figure 7. It is seen that the trend is similar to that of the flexural strength.

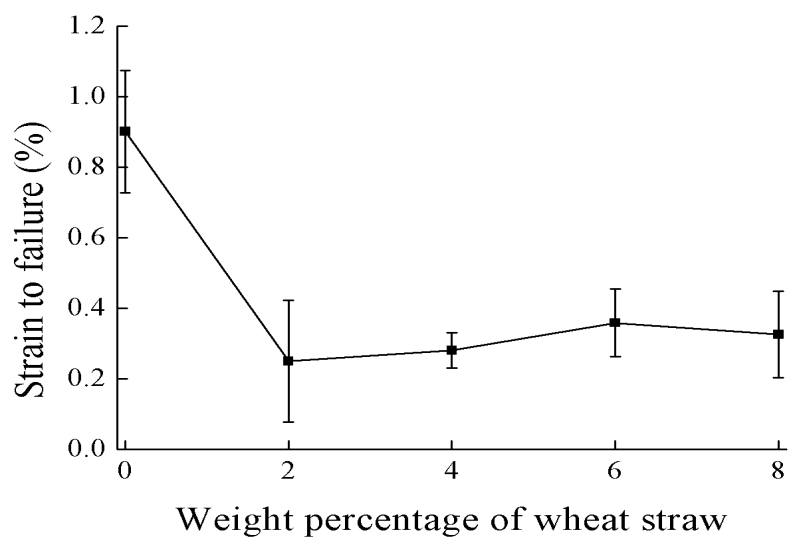

Figure 7. Strains to failure vs. weight percentage of wheat straw

\section{Conclusions}

The flexural properties of wheat straw reinforced polyester composites were studied. Tests were conducted in the three point bend configuration in accordance with ASTM D790-07 at a span to depth ratio of 16 . Four weight fractions of wheat straw $2 \%, 4 \%, 6 \%$ and $8 \%$ were chosen to be studied. The process-induced voids were studied and it is shown that voids play an important role in flexural properties. When voids exist, flexural modulus was calculated using a micromechanical model. It is shown from both the experimental results and calculation that flexural modulus decreases with the addition of wheat straw, and this reduction in flexural modulus is due to process-induced voids. Flexural strength of polyester resins does not increase with the addition of wheat straw as reinforcement. This is due to poor interfacial adhesion and the existence of voids. In order to achieve reinforcement, treatment of straw fibers and better processing techniques to reduce voids are needed. Although no promising results are shown from this study, it is shown that it is possible to recycle/reuse straw by incorporating it in polymer composites. 


\section{ACKNOWLEDGEMENTS}

The authors thank Sing Zhang Tie for carrying out all the experiments. Chensong Dong thanks the support from the Curtin Research Fellowship.

\section{REFERENCES}

[1] P. A. Fowler, J. M. Hughes and R. M. Elias, 2006, Biocomposites: technology, environmental credentials and market forces, Journal of the Science of Food and Agriculture, 86(12), 1781-1789

[2] C. Nyambo, A. K. Mohanty and M. Misra, 2010, Polylactidebased renewable green composites from agricultural residues and their hybrids, Biomacromolecules, 11(6), 1654-1660

[3] N. M. White and M. P. Ansell, 1983, Straw-reinforced polyester composites, Journal of Materials Science, 18(5), $1549-1556$

[4] S. Mishra and M. Sain, 2009, Commercialization of wheat straw as reinforcing filler for commodity thermoplastics, Journal of Natural Fibers, 6(1), 83-97
[5] S. r. Halvarsson, H. k. Edlund and M. Norgren, 2008, Properties of medium-density fibreboard (MDF) based on wheat straw and melamine modified urea formaldehyde (UMF) resin, Industrial Crops and Products, 28(1), 37-46

[6] Fibreglass \& Resin Sales Pty Ltd SR250 orthophthalic polyester resin data sheet

[7] D. Ray and J. Rout, 2005 Thermoset biocomposites. in A. K. Mohanty, M. Misra and L. T. Drzal (Eds.) Natural Fibers, Biopolymers, and Biocomposites, CRC Press

[8] ASTM International, 2007, Standard test methods for flexural properties of unreinforced and reinforced plastics and electrical insulating materials, D790, West Conshohocken, ASTM International

[9] M. Wollerdorfer and H. Bader, 1998, Influence of natural fibres on the mechanical properties of biodegradable polymers, Industrial Crops and Products, 8(2), 105-112

[10] E. H. Kerner, 1956, The elastic and thermo-elastic properties of composite media, Proceedings of the Physical Society, Section B, 69(8), 808

[11] P. K. Mallick, 1993, Fiber-Reinforced Composites: Materials, Manufacturing, and Design, New York, Marcel Dekker 